\title{
Prescrição eletrônica para a segurança na terapia medicamentosa: uma revisão integrativa
}

\author{
Eletronic prescription for safety in drug therapy: an integrative review
}

Receta electrónica para la seguridad em la terapia con drogas: una revisión integrativa

Ludimila Domingues Barbosa $^{1 *}$, Patricia Reis Alves dos Santos ${ }^{1}$.

\begin{abstract}
RESUMO
Objetivo: Este estudo objetivou investigar evidências científicas acerca do uso da Prescrição Eletrônica (PE) e do impacto desta conduta para a segurança na terapia medicamentosa. Métodos: Trata-se de Revisão Integrativa, tendo sido selecionados estudos publicados na última década, nos idiomas português, espanhol e inglês, localizados nas bases de dados: MEDLINE via PubMed, CINAHL, Biblioteca Virtual em Saúde (BVS) e LILACS. Utilizou-se o software Rayyan para a extração dos estudos encontrados. Resultados: Sete artigos constituíram a amostra, $86 \%$ deles publicados nos últimos 5 anos e desenvolvidos no cenário hospitalar. 0 uso da PE proporcionou redução dos erros na terapia medicamentosa, diminuição na ausência de dados e eliminação de ilegibilidade e de abreviações. Entretanto, o sistema eletrônico não é a única solução para prevenir erros de medicação, pois outros fatores devem ser considerados, tais como conhecimento dos prescritores sobre os medicamentos, tipos de medicamentos prescritos, estresse e comunicação. Dentre as ameaças, destacaram-se a não valorização dos profissionais quanto aos alertas gerados pelo sistema e a possibilidade de novos erros. Considerações finais: A PE mostrou-se mais segura em relação à prescrição manual, mas, se não utilizada adequadamente, pode representar fator de risco para a ocorrência de erros mais graves.
\end{abstract}

Palavras-chave: Segurança do paciente, Prescrição eletrônica, Serviços de saúde, Tecnologia.

\begin{abstract}
Objective: This study aims to "Investigate the scientific evidence about the use of Electronic Prescription (EP) and safety in drug therapy". Methods: The integrative review method was used, selected studies from the last decade, in Portuguese, Spanish and English, with databases: MEDLINE via PubMed, CINAHL, Virtual Health Library and LILACS. He used the Rayyan software to extract the studies found. Results: Of the total number of articles, seven constituted the sample. It was observed that $86 \%$ were published in the last 5 years and developed in the hospital setting. The use of EP evidenced the reduction of errors in drug therapy, the reduction in the absence of data, the elimination of illegibility and abbreviations. However, the electronic system is not the only solution, and other aspects must be considered, such as: the knowledge of prescribers, the types of medication prescribed, stress and communication. Among the threats, professionals do not appreciate the alerts generated by the system and the possibility of new errors. Final considerations: It proved to be safer compared to manual prescription, however it awakens to the fact that if not used properly, it may represent a risk factor for the occurrence of more serious errors.
\end{abstract}

Keywords: Patient safety, Electronic prescription, Health services, Technology.

\section{RESUMEN}

Objetivo: Este estudio tiene como objetivo "Investigar la evidencia científica sobre el uso de la Receta Electrónica (RE) y la seguridad en la farmacoterapia". Métodos: Se utilizó el método de revisión integradora, estudios seleccionados de la última década, en portugués, español e inglés, con bases de datos: MEDLINE vía PubMed, CINAHL, Virtual Health Library y LILACS. Utilizó el software Rayyan para extraer los estudios encontrados. Resultados: Del total de artículos, siete constituyeron la muestra. Se observó que $86 \%$ se publicaron en los últimos 5 años y se desarrollaron en ámbito hospitalario. El uso de RE evidenció la reducción

${ }^{1}$ Centro Universitário Municipal de Franca (Uni-FACEF), Franca - SP. *E-mail: ludimiladbarbosa@gmail.com

Financiado pelo Programa de Iniciação Científica (Programa IC Uni-FACEF), Processo 2019-2020 
de errores en farmacoterapia, la reducción en ausencia de datos, la eliminación de ilegibilidad y abreviaturas. Sin embargo, el sistema electrónico no es la única solución, debiéndose considerar otros aspectos: el conocimiento de los prescriptores, los tipos de medicamentos prescritos, el estrés y la comunicación. Entre las amenazas, los profesionales no aprecian las alertas que genera el sistema y la posibilidad de nuevos errores. Consideraciones finales: Resultó ser más seguro en comparación con la prescripción manual, sin embargo, se da cuenta de que si no se usa correctamente, puede representar un factor de riesgo para la ocurrencia de errores más graves.

Palabras clave: Seguridad del paciente, Prescripción Electrónica, Asistencia Sanitaria, Tecnología

\section{INTRODUÇÃO}

A segurança do paciente é um dos elementos essenciais para a qualidade do cuidado em saúde. Tratase, segundo a Organização Mundial da Saúde (OMS), da redução, a um mínimo aceitável, do risco de dano desnecessário associado a esse cuidado (WORLD HEALTH ORGANIZATION (WHO), 2009).

As falhas decorrentes do cuidado em saúde geram prejuízos imensuráveis a todos os envolvidos, uma vez que há significativo aumento do custo, danos físicos e psíquicos ao paciente e também aos profissionais, além de eventual perda de vidas humanas (ULLSTRÖM S, et al., 2013; VILELA RPB, et al., 2018).

Com base nos dados cadastrados pelas instituições de saúde no NOTIVISA (Sistema de Notificações em Vigilância Sanitária), de junho de 2019 a maio de 2020, foram notificados 151.442 incidentes associados à assistência à saúde, dos quais 38,9\% ocorreram na Região Sudeste (AGÊNCIA NACIONAL DE VIGILÂNCIA SANITÁRIA, 2020). Os erros no processo da terapia medicamentosa representam uma parcela importante dessas ocorrências.

Entende-se por erro de medicação qualquer evento evitável relacionado ao uso de medicamentos nas etapas de prescrição, dispensação, preparo, administração e monitoramento, seja ocasionado pelo profissional de saúde ou paciente (WHO, 2009). Dentre os erros mais comuns, podemos citar: prescrição não realizada ou atrasada, administração de medicamento a qual paciente é alérgico, erros de dose, trocas de via de administração (GIMENES FRE, et al., 2011; TEIXEIRA TCA e CASSIANI SHB, 2014)

Estima-se que 1,5 milhões de eventos adversos evitáveis relacionados à medicação ocorrem a cada ano nos Estados Unidos, com pacientes hospitalizados sofrendo, ao menos, 400.000 eventos adversos por ano. Os custos hospitalares para tratamento de eventos adversos e erros de medicação em pacientes internados chegam a aproximadamente $\$ 3.5$ bilhões em todo o país. Em ambulatórios, o custo anual associado à morbidade e mortalidade causadas por eventos de medicação nos Estados Unidos foi estimado em US\$177.4 bilhões (VILELA RPB, 2016; WHO, 2017).

Com o reconhecimento do risco elevado que existe em danos associados a administração de medicamentos, em 2017, a OMS lançou o terceiro Desafio Global de Segurança do Paciente com o tema "Medicação sem Danos". Com o objetivo de reduzir pela metade os danos graves e evitáveis relacionados a medicação, ao longo dos próximos cinco anos, a partir do avanço dos sistemas de saúde mais seguros e eficientes na assistência do paciente e nas etapas do processo de medicação (WHO, 2017).

A terapia medicamentosa apresenta várias etapas, incluindo prescrição, dispensação, preparo, administração e monitoramento pós-administração. Esse processo torna-se complexo pois compreende etapas que são delegadas a uma equipe multidisciplinar. Sendo das etapas de preparo e administração mais comumente executadas pela equipe de enfermagem, quando na ocorrência de erros de medicação, estes são frequentemente associados a esses trabalhadores, ainda que se saiba que esses ocorridos podem acontecer por situações referentes ao trabalho e a processos em não conformidades (SANTOS PRA, et al., 2019; TEIXEIRA TCA e CASSIANI SHB, 2014).

No que concerne a eventos adversos envolvendo terapia medicamentosa, estudos têm evidenciado erros frequentes na etapa de dispensação de medicamentos e no processo de preparo e administração (TEIXEIRA TCA e CASSIANI SHB, 2014). Entretanto, algumas ações são efetivas para minimizar eventos adversos 
relacionados a medicamentos, a exemplo dos checklists, protocolos para a prática e utilização de sistemas de Prescrição Eletrônica (PE), favorecendo a diminuição da distração e prevenindo erros (MENEGUETI MG, et al., 2017; TEIXEIRA TCA e CASSIANI SHB, 2014).

A PE é um tipo de tecnologia que compreende diversos softwares, desenvolvidos para prescrever medicamentos de forma automatizada, constituindo-se de um formato padrão, legível e com integralidade de informações necessárias para a prescrição segura de medicamentos (CARDOSO AM, 2013).

Quanto às principais vantagens da PE em relação aos manuais, tem sido discutida atualmente a segurança de uma prescrição legível e com conteúdo necessário para a realização da terapia medicamentosa de forma segura. Também é possível com a PE implementar ferramentas de apoio a decisão clínica e alertas de interações medicamentosas (PERDIGÃO P, et al., 2014; KAWANO DF, et al., 2006).

No entanto, a PE não pode ser vislumbrada como a única solução para prevenção dos erros de medicação, e sim como uma das estratégias possíveis, uma vez que se não for corretamente utilizada pode, inclusive, contribuir para a ocorrência de novos erros (GIMENES FRE, et al., 2011; ROSA MBR, et al., 2019).

Práticas seguras no processo de medicação requerem dos profissionais sensibilidade e atenção para o planejamento e implementação de protocolos a curto, médio e longo prazo, em busca de prevenir e reduzir potenciais para erros. Ressalta-se que a colaboração de uma equipe multidisciplinar é peça-chave para 0 sucesso dessas ações (SANTOS PRA, et al., 2019).

Diante desta problemática, este estudo buscou conhecer quais são as evidências científicas disponíveis sobre o uso da prescrição eletrônica para a mitigação de erros relacionados à terapia medicamentosa, no contexto dos serviços de saúde. Como objetivo, estabeleceu-se: investigar as evidências científicas acerca do uso da PE e do impacto desta conduta para minimização de erros na terapia medicamentosa, no contexto dos serviços de saúde.

\section{MÉTODOS}

Este estudo utilizou como método de pesquisa a Revisão Integrativa da literatura, em busca de apreender o conhecimento produzido, na última década, acerca do uso da prescrição eletrônica e do impacto dessa estratégia para a mitigação de erros relacionados à terapia medicamentosa.

A Revisão Integrativa é um método de pesquisa que possibilita a busca, a avaliação crítica e a síntese do conhecimento produzido acerca de determinado tema, contribuindo para a atualização e identificação de lacunas, subsidiando futuros estudos (MENDES KDS, et al., 2008).

Para o desenvolvimento deste estudo foram percorridas as seguintes etapas: Etapa 1: Elaboração da questão de pesquisa; Etapa 2: Seleção das palavras-chave e estratégia de busca; Etapa 3: Busca de estudos nas bases de dados; Etapa 4: Coleta de dados; Etapa 5: Análise crítica dos estudos.

Para elaboração da questão de pesquisa e seleção dos descritores foi utilizada a estratégia PICO (P referese ao Paciente ou Problema; a letra I à Intervenção; a $\mathrm{C}$ ao Controle ou Comparação; e a letra O refere-se à Outcome, traduzido como desfecho ou resultados). Adotou-se a seguinte questão norteadora: Quais são as evidências acerca do uso da prescrição eletrônica para a mitigação de erros relacionados à terapia medicamentosa, no contexto dos serviços de saúde?

A estratégia de busca deu-se pela combinação dos descritores ("patient safety" AND "Medication Errors "AND" Electronic Prescribing").

A busca dos estudos primários ocorreu nos meses de maio e junho de 2020. Foram consultadas as bases de dados: MEDLINE via PubMed da National Library of Medicine, Cumulative Index to Nursing and Allied Health Literature (CINAHL), Biblioteca Virtual em Saúde (BVS) e Literatura Latino-Americana e do Caribe em Ciências da Saúde (LILACS).

Constituíram critérios de inclusão para seleção e elegibilidade: artigos disponíveis na íntegra (texto completo e gratuito); nos idiomas inglês, espanhol e português; publicados no período de 2010 a junho de 
2020; desenvolvidos nos cenários de serviços de saúde (atenção primária, serviços de urgência, serviços hospitalares, serviço ambulatorial); com a população adulta e com foco no uso da prescrição eletrônica para fortalecer a segurança do paciente. Foram excluídos estudos de revisão, comentários, resumos, cartas ao editor e editoriais.

$\mathrm{Na}$ etapa de coleta de dados, utilizou-se o software Rayyan para a extração dos estudos encontrados nas bases de dados. O Rayyan é um aplicativo habilitado para web que proporciona mais agilidade aos autores de revisões sistemáticas durante a triagem inicial de resumos e títulos usando um processo de semiautomação (OUZZANI M, et al., 2016).

Por meio dessa ferramenta, os autores importam os arquivos das bases de dados, carregando uma lista de estudos obtidos, e começam a classificá-los para inclusão e exclusão. Além do pesquisador principal, outro integrante teve acesso ao Rayyan para realizar a triagem inicial, de forma cegada. Os artigos que apresentaram conflito de elegibilidade entre os dois pesquisadores passaram pela análise e elegibilidade do professor orientador, totalizando a seleção de 7 estudos. A baixa quantidade de artigos deu-se pela razão de que muitos estudos não estavam disponíveis na íntegra de forma gratuita, sendo um dos critérios de seleção, assim como a não tinha correlação com a proposta da revisão.

Para análise e síntese dos resultados dos artigos em sua íntegra, elaborou-se uma planilha no Microsoft Excel, a qual contemplava informações para identificação do artigo, como ano, país, autores, periódico, características metodológicas e avaliação dos resultados e discussão.

A análise desses dados compôs os resultados e a discussão da presente revisão. A seguir, apresenta-se o fluxograma com as etapas percorridas nesta investigação para o alcance dos resultados (Figura 1):

Figura 1 - Fluxograma do processo de seleção dos estudos.

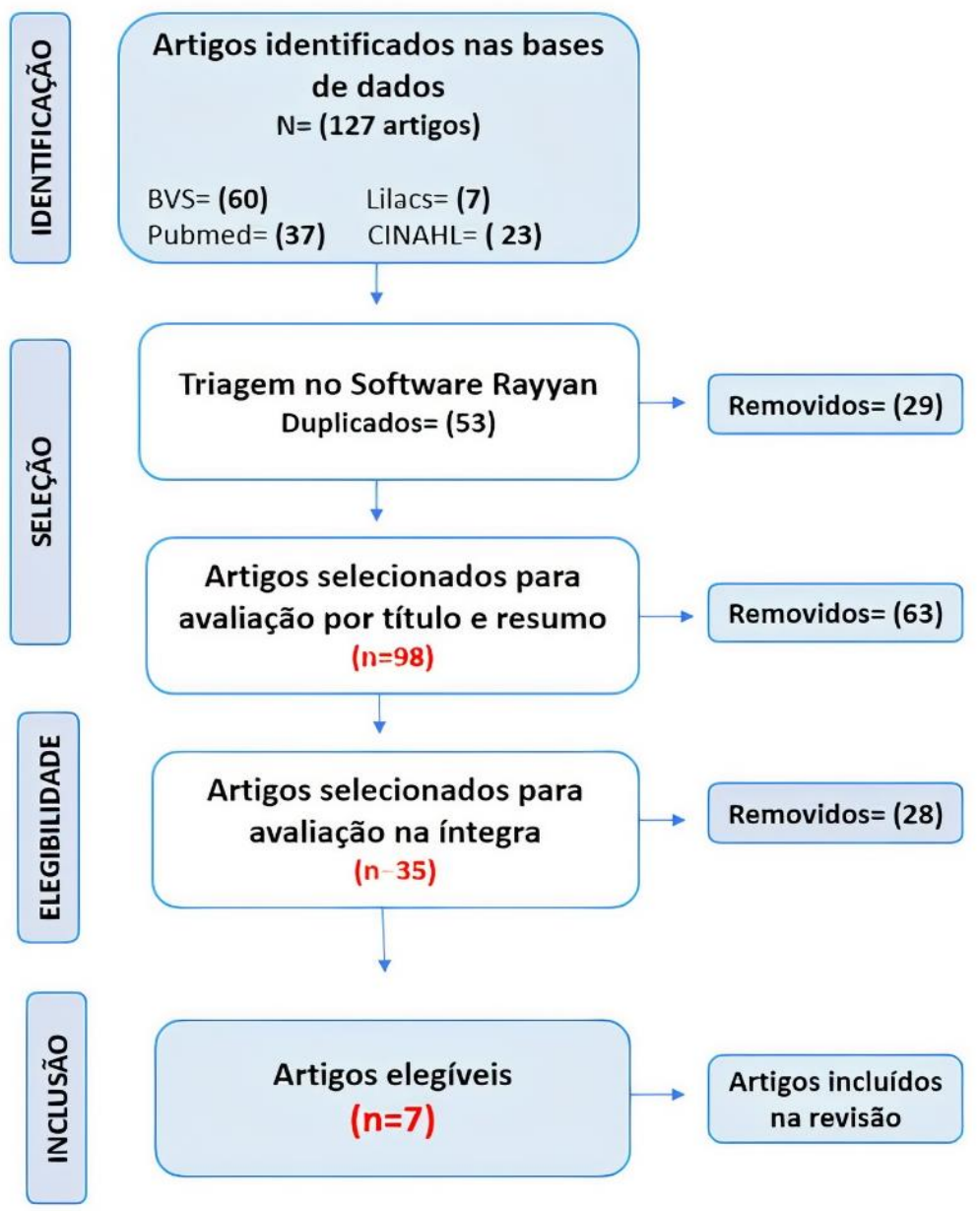

Fonte: Barbosa LD e Santos PRA, 2021. 


\section{RESULTADOS}

Do total de artigos encontrados nas bases de dados, sete (7) constituíram a amostra para a síntese qualitativa, após aplicação dos critérios de inclusão e exclusão. Apresentam-se, a seguir, os estudos analisados segundo autor, país, periódico, ano, objetivos e cenário do estudo (Quadro 1):

Quadro 1 - Caracterização dos estudos segundo autor, periódico, país, ano, objetivos e cenário do estudo.

\begin{tabular}{|c|c|c|c|c|}
\hline & Título & Autor\País\Periódico\Ano & Objetivos & $\begin{array}{l}\text { Cenário } \\
\text { do } \\
\text { estudo }\end{array}$ \\
\hline A & $\begin{array}{c}\text { The impact of electronic } \\
\text { prescription on reducing } \\
\text { medication errors in an } \\
\text { Egyptian outpatient } \\
\text { clinic. }\end{array}$ & $\begin{array}{c}\text { KENAWY A S e KETT } \\
\text { VRevista Internacional de } \\
\text { Informática } \\
\text { MédicalEgitol2019. }\end{array}$ & $\begin{array}{l}\text { Avaliar o impacto da prescrição } \\
\text { eletrônica nos erros de } \\
\text { medicação nas fases de } \\
\text { prescrição e dispensação e } \\
\text { formular recomendações sobre o } \\
\text { uso de prescrições eletrônicas } \\
\text { na prática ambulatorial egípcia. }\end{array}$ & Hospital \\
\hline B & $\begin{array}{l}\text { Electronic prescription: } \\
\text { frequency and severity } \\
\text { of medication errors }\end{array}$ & $\begin{array}{c}\text { ROSA M B, et al.\Revista } \\
\text { da Associação Médica } \\
\text { Brasileira\Brasil } 2019 .\end{array}$ & $\begin{array}{l}\text { Avaliar a frequência e a } \\
\text { gravidade dos erros de } \\
\text { prescrição com medicamentos } \\
\text { potencialmente perigosos, antes } \\
\text { e após a introdução de um } \\
\text { sistema computadorizado de } \\
\text { entrada de pedidos de } \\
\text { provedores (CPOE). }\end{array}$ & Hospital \\
\hline C & $\begin{array}{c}\text { Patient-centric } \\
\text { implementation of an } \\
\text { electronic medication } \\
\text { management system at } \\
\text { a tertiary hospital in } \\
\text { Western Sydney. }\end{array}$ & $\begin{array}{l}\text { GUNJA N, et al. } \backslash \text { Journal } \\
\text { of innovation in health } \\
\text { informatics } \backslash \text { Australia } \\
2018 .\end{array}$ & $\begin{array}{l}\text { Descrever a lógica, o método e a } \\
\text { implementação de um novo } \\
\text { modelo na conversão do papel } \\
\text { para a PE, centrado no paciente. }\end{array}$ & Hospital \\
\hline D & $\begin{array}{l}\text { Audit on the Use of } \\
\text { Dangerous } \\
\text { Abbreviations, } \\
\text { Symbols, and Dose } \\
\text { Designations in Paper } \\
\text { Compared to Electronic } \\
\text { Medication Orders: A } \\
\text { Multicenter Study. }\end{array}$ & $\begin{array}{l}\text { CHEUNG S, et al. } \backslash \text { Ann } \\
\text { Pharmacotherl. } \\
\text { Canadál2018. }\end{array}$ & $\begin{array}{l}\text { Comparar as taxas de uso de } \\
\text { abreviações perigosas da lista } \\
\text { "Não usar", em pedidos de } \\
\text { medicamentos em papel e } \\
\text { eletrônicos. E também } \\
\text { determinar a proporção de } \\
\text { pacientes sob risco de erros de } \\
\text { medicação e as abreviações } \\
\text { perigosas mais usadas. }\end{array}$ & Hospital \\
\hline$E$ & $\begin{array}{l}\text { Medication errors: } \\
\text { electronic vs. paper- } \\
\text { based prescribing. } \\
\text { Experience at a tertiary } \\
\text { care university hospital. }\end{array}$ & $\begin{array}{l}\text { HINOJOSA-AMAYA J M, } \\
\text { et al. YJournal Evaluation } \\
\text { in Clinical Practice } 2016 \text {. }\end{array}$ & $\begin{array}{l}\text { Comparar os erros de } \\
\text { medicação entre a prescrição } \\
\text { eletrônica e em papel detectados } \\
\text { durante a farmacovigilância. }\end{array}$ & Hospital \\
\hline $\mathrm{F}$ & $\begin{array}{l}\text { Risk factors for } \\
\text { medication errors in the } \\
\text { electronic and manual } \\
\text { prescription }\end{array}$ & $\begin{array}{l}\text { VOLPE CRG. et al. } \\
\text { \Revista Latino- } \\
\text { Americana de } \\
\text { Enfermagem\Brasil|2016 }\end{array}$ & $\begin{array}{l}\text { Comparar as prescrições } \\
\text { eletrônicas e manuais de um } \\
\text { hospital público do Distrito } \\
\text { Federal, identificando os fatores } \\
\text { de risco para ocorrência de erros } \\
\text { de medicação. }\end{array}$ & Hospital \\
\hline G & $\begin{array}{l}\text { Electronic prescribing } \\
\text { increases uptake of } \\
\text { clinical } \\
\text { pharmacologists' } \\
\text { recommendations in the } \\
\text { hospital setting. }\end{array}$ & $\begin{array}{l}\text { TAEGTMEYER AB, et al. } \\
\text { \British Journal of Clinical } \\
\text { Pharmacology \Suiçal } \\
2011 .\end{array}$ & $\begin{array}{l}\text { Estudar o impacto da prescrição } \\
\text { eletrônica na aceitação das } \\
\text { sugestões de farmacologistas } \\
\text { clínicos em ambiente hospitalar. }\end{array}$ & Hospital \\
\hline
\end{tabular}

Fonte: Barbosa LD e Santos PRA, 2021. 
Dos artigos incluídos nesta revisão, dois foram realizados no Brasil (28,5\%), e os demais na Suíça, Canadá, Austrália e Egito. Em relação ao ano, a maioria (86\%) foi publicada nos últimos 5 anos. Quanto ao contexto de implementação e uso da PE, todos foram realizados no cenário hospitalar.

Em relação ao uso da PE para o fortalecimento da segurança na terapia medicamentosa e redução dos erros nesse processo, foram enumeradas diversas fortalezas. No entanto, também foram apontadas ameaças associadas ao sistema eletrônico de prescrição e, também, relacionadas ao uso dos sistemas pelos profissionais.

Destaca-se a ausência de dados nas PE e a não valorização pelos profissionais quanto aos alertas gerados pelo sistema eletrônico. Tais situações contribuíram para a ocorrência de erros mais graves, bem como para a introdução de novos erros.

\section{DISCUSSÃO}

Sabe-se que a PE pode contribuir de forma relevante para a segurança do paciente no processo da terapia medicamentosa. Entretanto, alguns fatores causais relacionados aos erros de medicação podem não ser abolidos somente com o uso desta estratégia, reconhecida e recomendada internacionalmente, assim como ela própria (a PE) pode estar relacionada com a possibilidade de novos erros (ROSA MB, et al., 2019).

Na presente revisão foi evidenciado que o sistema eletrônico eliminou alguns erros, como caligrafia pouco clara e aspectos legais das prescrições, por exemplo dados do paciente (KENAWY AS e KETT V, 2019). Tal como apontado por Volpe CRG, et al. (2016), com a PE a ilegibilidade é um fator de risco eliminado, e oferece ao profissional um apoio para sua decisão clínica, promovendo clareza na comunicação entre os profissionais e exatidão de informações, com o objetivo final de aprimorar a prescrição.

Ressalta-se que possíveis dificuldades encontradas pelos farmacêuticos, enfermeiros e técnicos de enfermagem, na compreensão das medicações e orientações prescritas de forma manuscrita, podem dificultar a atuação desses profissionais. Podem, também, potencializar o risco de erros de medicação, principalmente nas etapas de dispensação, preparo e administração, comprometendo a segurança do paciente (VOLPE CRG, et al., 2016).

Quanto ao uso de abreviações, a PE mostrou-se estratégica para a redução significativa das taxas de uso de abreviaturas perigosas, em comparação às prescrições de medicamentos em papel (CHEUNG S, et al., 2018). O uso de abreviaturas não é aconselhado na prescrição de medicamentos visto que isso pode elevar a chance de ocorrer algum erro de medicação. A elaboração e divulgação de uma lista de abreviaturas, padronizadas pela própria instituição, é uma alternativa viável caso seja imprescindível a abreviação. Isso tornará a comunicação mais efetiva entre a equipe (BRASIL, 2013; WHO, 2017).

Quando se utiliza a PE, um aspecto positivo é a presença de itens indispensáveis na prescrição, como via de administração, diluição e frequência da medicação. É significativo destacar que a PE, por ser mais completa e organizada, possibilitou a redução da frequência de erros graves, principalmente no momento do preparo, dispensação e administração do medicamento ao paciente (ROSA MBR, et al., 2019; VOLPE CRG, et al., 2016).

Associa-se o uso da PE à redução de alguns erros de medicação, por exemplo escrita incorreta do médico ou medicamentos parecidos. Por conseguinte, nota-se maior número de prescrições sem erros, diferentemente do que se observa no sistema de prescrição manual (HINOJOSA- AMAYA JM, et al., 2016).

No entanto, apesar das vantagens apresentadas pelo sistema de prescrição eletrônica em relação à prescrição manual, o estudo de Rosa MBR, et al. (2019) mostrou o potencial da PE em aumentar o número de erros e eventos adversos durante os primeiros anos após sua implantação. Isso porque falhas no desenvolvimento são detectadas e corrigidas somente após determinado período de uso dos sistemas pelos profissionais.

A PE apresenta-se vulnerável a processos propensos a erros, e mesmo que os sistemas de tecnologias em informação possam trazer benefícios para a assistência à saúde, ainda podem ser capazes de ocasionar novos tipos de riscos, contribuindo para danos ao paciente (JIA Y, et al., 2019). 
Estudo mostrou que até $91 \%$ das mensagens de alertas geradas pelo sistema eletrônico de prescrição foram ignoradas pelos médicos no momento da prescrição, sendo importante investigar o motivo da não adesão a essas intervenções sugeridas (TAEGTMEYER AB, et al., 2019).

Os farmacêuticos participantes do estudo de Redwood S, et al. (2011) relataram ter recebido PE erradas diariamente porque os prescritores selecionaram informações incorretas, como medicamento errado, paciente errado, forma ou dosagem incorreta. Se isso não fosse percebido na farmácia, o paciente poderia sofrer repercussões negativas.

Houve diferenças em relação à avaliação da implementação da PE nos diversos setores das Instituições Hospitalares. No estudo de Kenawy AS e Kett V (2019), a prescrição eletrônica teve o poder de reduzir as taxas de erro de prescrição apenas nos departamentos de pediatria e neurologia e falhou em reduzir a taxa nos outros departamentos ambulatoriais.

Nesta revisão, as fortalezas observadas quanto ao uso da PE atentam-se para o fato dessa ser capaz de assegurar uma prescrição mais segura na terapia medicamentosa, consequentemente contribuir para a segurança do paciente. No entanto, pode não alcançar seus objetivos quando não aplicada corretamente, como não eliminar a possibilidade de erros de medicação, além de representar fator de risco para ocorrência de erros graves e até mesmo para a introdução de novos erros, tornando-se uma ameaça à segurança na terapia medicamentosa (HINOJOSA-AMAYA JM, et al., 2016; VOLPE CRG, et al., 2016; ROSA MB, et al., 2019).

Os resultados deste estudo também evidenciaram que o sistema eletrônico não é a única solução para o problema dos erros de medicação, e devem ser considerados outros aspectos, como conhecimento dos prescritores, número e tipos de medicamentos prescritos, estresse e comunicação. Nessa perspectiva, os profissionais de saúde devem estar diretamente envolvidos no desenvolvimento e implementação do sistema eletrônico, com o propósito de projetarem sistemas mais seguros e eficientes. Sugere-se que a implantação seja feita quando os hospitais e suas equipes se sentirem confortáveis e habituados para o uso do sistema (GUNJA N, et al., 2018).

Demonstrou-se que aspectos específicos do software e hardware (como tamanho da tela, menus suspensos e ordem das escolhas) podem contribuir para a seleção de doses e medicamentos errados. Para a implementação de melhores práticas, faz-se necessário identificar e compreender as relações dos erros com características do software e do hardware utilizados para as prescrições, de modo a identificar falhas de usabilidade potencialmente perigosas (LAPANE KL, et al., 2011).

Estudo demonstrou satisfação dos profissionais médicos com o potencial do uso de sistemas no sentido de facilitar os processos de prescrição em sua prática. No entanto, destacaram vários problemas e áreas sujeitas a adequações, visto que algumas das principais funções do processo de prescrição não funcionavam corretamente, como exemplo, a correção e o cancelamento das prescrições eletrônicas (KAUPPINEN H, et al., 2017).

Nesse sentido, acredita-se que os recursos de design da interface dos sistemas de prescrição eletrônica podem estar relacionados à usabilidade dos sistemas e à segurança da prescrição. A criação de um design centrado no usuário em sistemas eletrônicos permite que os participantes interajam e trabalhem de maneira eficiente e segura, além de possibilitar um processo padronizado de prescrição (AUFEGGER L, et al., 2020).

Possíveis soluções foram propostas por Syed-Abdul S, et al. (2014). Os autores recomendaram a criação de um modelo inteligente de decisão terapêutica na escolha dos medicamentos para prescrições eletrônicas e que o sistema eletrônico fosse capaz de exibir uma lista de medicamentos relevantes que podem estar relacionados a uma determinada doença descrita pelo profissional. Acredita-se que esse modelo possa reduzir o tempo gasto pelos médicos para prescrever medicamentos e também eliminar o risco de erros de medicação, mesmo que os médicos selecionem acidentalmente o medicamento errado, uma vez que apenas os medicamentos mais relevantes para o caso estariam listados no menu.

É importante que os serviços de saúde incluam estratégias de prevenção aos sistemas eletrônicos de prescrição, tais como: alertar a forma de prescrição quando do uso de medicamentos parecidos ou similares; 
alertar interações perigosas encontradas no medicamento prescrito ou possíveis reações adversas graves a medicamentos; adicionar bloqueios de segurança, como verificação dupla de medicamentos de alto risco e o uso de código de barras na rotulação de medicamentos (HINOJOSA AMAYA JM, et al., 2016).

Ficou evidente a preocupação com o uso da PE no cenário hospitalar. Destaca-se a importância de estudos em outros cenários, por exemplo, em serviços de urgência e emergência, locais considerados de alto risco para erros de medicação. Acredita-se que a implementação de sistemas informatizados de prescrição nesses cenários pode contribuir para a diminuição de erros de medicação (SANTOS PRA, et al., 2019; OLIVEIRA RC, et al., 2005).

\section{CONSIDERAÇÕES FINAIS}

Este estudo identificou publicações a respeito do uso da PE e do impacto desta conduta para terapia medicamentosa. Evidenciou-se que $86 \%$ dos estudos foram publicados nos últimos 5 anos, em cenário hospitalar (100\%) e abordava fortalezas e ameaças no uso da PE na mitigação de erros. Dentre as fortalezas, destaca-se que a PE eliminou erros, como ilegibilidade, possibilitou a presença de informações essenciais na prescrição e redução no uso de abreviaturas. Dentre as ameaças destaca-se questões relacionadas ao uso inadequado dos sistemas pelos profissionais, como, a não adesão de alertas gerados pelo sistema. O sistema eletrônico não é a única solução para o problema, e deve ser considerado o conhecimento dos prescritores, número e tipos de medicamentos prescritos, estresse, comunicação e usabilidade do sistema.

\section{AGRADECIMENTOS E FINANCIAMENTO}

Agradecemos ao Centro Universitário Municipal de Franca - Uni-FACEF, pelo apoio e financiamento desta pesquisa por meio do Programa de Iniciação Científica (Programa IC Uni-FACEF).

\section{REFERÊNCIAS}

1. AGÊNCIA NACIONAL DE VIGILÂNCIA SANITÁRIA (ANVISA). Boletim Informativo Segurança do Paciente e Qualidade em Serviços de Saúde. Incidentes relacionados à assistência à saúde Resultados das notificações realizadas no Notivisa - São Paulo, junho de 2019 a maio de 2020. Disponível em: https://www20.anvisa.gov.br/segurancadopaciente/index.php/publicacoes/category/relatorios-dos-estados>. Acesso em: 05 jul. 2020.

2. AUFEGGER L, et al. Evaluating users' experiences of electronic prescribing systems in relation to patient safety: a mixed methods study. BMC Med Inform Decis Mak. 2020; 20(1): 62.

3. CARDOSO AM. Implantação de prescrição eletrônica afim de otimizar a dispensação de medicamentos. Revista Brasileira de Farmácia Hospitalar e Serviços de Saúde, 2013; 4(4): 39-45.

4. CHEUNG S, et al. Audit on the Use of Dangerous Abbreviations, Symbols, and Dose Designations in Paper Compared to Electronic Medication Orders: A Multicenter Study. Annals of Pharmacotherapy, 2018; 52(4).

5. GIMENES FRE, et al. Administração de medicamentos, em vias diferentes das prescritas, relacionada à prescrição médica. Revista Latino-Americana de Enfermagem, 2011; 19(1): 11-17.

6. GUNJA N, et al. Patient-centric implementation of an electronic medication management system at a tertiary hospital in Western Sydney. JOURNAL OF INNOVATION IN HEALTH INFORMATICS, 2018; 25(3): 169-175.

7. HINOJOSA-AMAYA JM, et al. Medication errors: electronic vs. paper-based prescribing. Experience at a tertiary care university hospital. Journal of Evaluation in Clinical Practice, 2016; 22(5): 751 - 754.

8. JIA Y, et al. Developing a Safety Case for Electronic Prescribing. Stud Health Technol Inform. 2019; 264: 629-633.

9. KAUPPINEN H, et al. Medication safety and the usability of electronic prescribing as perceived by physicians-A semistructured interview among primary health care physicians in Finland. J Eval Clin Pract. 2017; 23(6): 1187-1194.

10. KAWANO DF, et al. Acidentes com os medicamentos: como minimizá-los?. Revista Brasileira de Ciências Farmacêuticas, 2006; 42(4): 487-495.

11. KENAWY AS, KETT V. The impact of electronic prescription on reducing medication errors in an Egyptian outpatient clinic. International Journal of Medical Informatics, 2019; 127: 80-87.

12. LAPANE KL, et al. E-Prescribing and patient safety: Results from à mixed method study. Am J Pharm Benefits. 2011; $3(2): 24-34$.

13. MENDES KDS, et al. Revisão integrativa: método de pesquisa para a incorporação de evidências na saúde e na enfermagem. Texto \& Contexto - Enfermagem, 2008; 17(4): 758-764. 
14. MENEGUETI MG, et al. Erros no processo de medicação: Proposta de uma estratégia educativa baseada nos erros notificados. Revista de Enfermagem, 2017; 11(5): 2046-2055.

15. OLIVEIRA RC, et al. Estratégias para prevenção de erros na medicação no setor de emergência. Revista brasileira enfermagem, 2005; 58(4): 399-404.

16. OUZZANI M, et al. Rayyan - a web and mobile app for systematic reviews. Systematic Reviews, 2016; 5(1): 1-10.

17. PERDIGÃO P, et al. Erros relacionados a medicamentos. In: SOUSA P, MENDES W. (Org). Segurança do paciente: criando organizações de saúde seguras. Fiocruz, 2014; 2.

18. REDWOOD S, et al. Does the implementation of an electronic prescribing system create unintended medication errors? A study of the sociotechnical context through the analysis of reported medication incidents. BMC Medical Informatics and Decision Making, 2011; 29(11): 1-11.

19. ROSA MB, et al. Electronic prescription: frequency and severity of medication errors. Revista da Associação Médica Brasileira, 2019; 65(11): 1349-1355.

20. SANTOS PRA, et al. Ações para segurança na prescrição, uso e administração de medicamentos em unidades de pronto atendimento. Revista Gaúcha de Enfermagem, 2019; 40: 1-9.

21. SYED-ABDUL $S$, et al. A smart medication recommendation model for the electronic prescription. Comput Methods Programs Biomed, 2014; 117(2): 218-224.

22. TAEGTMEYER AB, et al. Electronic prescribing increases uptake of clinical pharmacologists' recommendations in the hospital setting. British Journal of Clinical Pharmacology, 2019; 72(6): 958 - 964.

23. TEIXEIRA TCA, CASSIANI SHB. Análise de causa raiz de acidentes por quedas e erros de medicação em hospital. Acta Paulista de Enfermagem, 2014; 27(2): 100-107.

24. ULLSTRÖM S, et al. Suffering in silence: a qualitative study of second victims of adverse events. BMJ: Quality \& Safety in Health Care, 2013; 10: 1-7.

25. VILELA RPB. Erro de medicação: o custo e o impacto das tecnologias preventivas na cadeia medicamentosa. Dissertação (Mestrado em Enfermagem) - Faculdade de Medicina de São José do Rio Preto, São José do Rio Preto, 2016; $185 \mathrm{p}$.

26. VOLPE CRG, et al. Risk factors for medication errors in the electronic and manual prescription. Revista LatinoAmericana de Enfermagem, 2016; 24: 1 - 9.

27. WORLD HEALTH ORGANIZATION (WHO). The Conceptual Framework for the International Classification for Patient Safety. 2009. Disponível em: http://www.who.int/patientsafety/implementation/taxonomy/publications/en/. Acesso em: 01 de julho de 2020

28. WORLD HEALTH ORGANIZATION (WHO). Medication Without Harm - Global Patient Safety Challenge on Medication Safety. Geneva: World Health Organization, 2017. Disponível em: https://www.who.int/initiatives/medication-withoutharm. Acesso em: 28 de junho de 2020. 